NK CELL HOMEOSTASIS AND CANCER IMMUNITY

DR JAI RAUTELA (Orcid ID : 0000-0002-4253-9966)

Article type : Special Feature Review

\title{
Molecular insight into targeting the NK cell immune response to cancer
}

Jai Rautela*, Fernando Souza-Fonseca-Guimaraes, Soroor Hediyeh-Zadeh, Rebecca B. Delconte, Melissa J. Davis and Nicholas D. Huntington

The Walter and Eliza Hall Institute of Medical Research, 1G Royal Parade, Parkville, Victoria 3052, Australia and Department of Medical Biology, University of Melbourne, Victoria 3050 Australia.

Melissa Davis davis.m@wehi.edu.au

Soroor Zadeh hediyehzadeh.s@wehi.edu.au

*Jai Rautela rautela.j@wehi.edu.au *Corresponding author

Nick Huntington huntington@wehi.edu.au

Fernando Souza-Fonseca-Guimaraes guimaraes.f@wehi.edu.au

Rebecca Deleonte delconte.r@wehi.edu.au

Keywords: NK cells, Cancer, Interleukins, IL-15, immune homeostasis, STAT5

Abstract

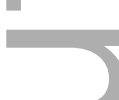

Abstract

Natural Killer (NK) cells have long been considered an important part of the anti-tumour immune response due to their potent cytolytic and cytokine-secreting abilities. To date, a clear This is the author manuscript accepted for publication and has undergone full peer review but has not been through the copyediting, typesetting, pagination and proofreading process, which may lead to differences between this version and the Version of Record. Please cite this article as doi: $\underline{10.1111 / \text { imcb. } 12045}$

This article is protected by copyright. All rights reserved 
demonstration of the role NK cells play in human cancer is lacking, and there are still very few examples of therapies that efficiently exploit or enhance the spontaneous ability of NK cells to destroy autologous cancer cells. Given the paradigm shift towards cancer immunotherapy over the past decade, there is a renewed push to understand how NK cell homeostasis and function are regulated in order to therapeutically harness these cells to treat cancer. This review will highlight recent advancements in our understanding of how growth factors impact on NK cell development, differentiation, survival and function with an emphasis on how these pathways may influence NK cell activity in the tumour microenvironment and control of cancer metastasis.

\section{IL-15 sets the boundaries of NK cell homeostasis in health and disease}

NK cells are derived from bone marrow progenitors and require the Inhibitor of DNA-binding protein 2 (ID2) for development ${ }^{1-6}$. Shortly following expression of Idb2 (encoding ID2) in NK progenitors, the IL-15 receptor complex is expressed and both IL- $15 \mathrm{R} \beta / \gamma$ and Idb2 are maintained at high levels during NK cell differentiation ${ }^{2,} 7$. Loss of ID2 function in NK cells significantly impairs their responsiveness to IL-15 most likely via upregulation of genes targets of ID2 binding proteins (E2A, E2-2 and HEB). As a consequence, NK cells are rapidly lost from peripheral tissues of mice following conditional deletion of $I d b 2^{2}$.

NK cell homeostasis is tightly controlled through an array of signal-transducing receptors that recognise soluble factors as well as cell-surface ligands. Interleukin-15 (IL-15) is the most crucial cytokine for permitting the development of NK cells from their bone-marrow progenitors. As a soluble cytokine, or 'trans-presented' in association with IL-15R $\alpha^{8}$, IL-15 triggers the IL15 receptor complex (IL-15R $\beta / \gamma$ encoded by $I l 2 r b$ and $I l 2 r g$ ) to change conformation and expose docking sites necessary for the subsequent JAK/STAT signalling cascade (Figure 2). The potency of this signalling pathway on the activation and proliferation of NK cells necessitates its strict control in order to maintain homeostatic function of this cell population.

The major way in which this control is exerted is through the restriction of IL-15 expression. Many stromal and haematopoietic cell types possess significant levels of the IL-15 transcript, though tight post-transcriptional regulation results in very low IL-15 protein levels in steadystate healthy conditions ${ }^{9}$. Following infection or other inflammatory stimuli such as tumour formation, IL-15 can be rapidly translated ${ }^{10-12}$. However, the fact that an IL-15-mediated 
accumulation of NK cells has never been reported in response to a biologically-relevant inflammatory stimulus highlights the constant scarcity and control exerted over this cytokine. The need for such tight control has been demonstrated by the dramatic immunopathology and NK cell expansion seen in mice bearing an IL-15 transgene under a ubiquitous promoter ${ }^{13}$. It appears therefore, that the dynamic range of IL-15 expression in vivo has been primarily set to maintain a precise number of NK cells, but also plays a secondary role during inflammation by promoting transient local NK cell activation.

IL-15 levels within a cancer may therefore be one of the major determinants of local NK cell activity and, by extension, patient outcome. Indeed, high IL-15 expression is associated with significantly better prognosis for melanoma (our unpublished data) as well as colorectal cancer patients, suggesting that the anti-tumour immune response (including $\mathrm{T}$ cells) is improved in the presence of IL-15 ${ }^{14}$. The obvious caveats to such studies are that transcript levels do not necessarily reflect protein levels, and that differences in local expression of IL-15R $\alpha$ strongly influence the biological activity of IL-15 ${ }^{15-19}$.

\section{IL-15 controls NK cell homeostasis via quality and quantity of STAT5 oligomers}

\section{STAT5 is necessary for appropriate IL-15 signalling}

The signal transducer and activator of transcription (STAT) family of proteins comprises 7 members; STAT1, 2, 3, 4, 5A, 5B and 6, and are found as inactive dimers in the cell cytoplasm. In NK cells, STAT5A and 5B play a central role in transducing the IL-15 signal. Following the ligation of the $\mathrm{LL}-15 \mathrm{R} \beta / \gamma$ complex, a cascade of reciprocal phosphorylation occurs resulting in the recruitment of JAK1 and JAK3 and the phosphorylation of both the receptor and JAK proteins ${ }^{20}$. Phosphotyrosine residues on the IL-15R $\beta / \gamma$ complex then provide docking sites for STAT5, enabling its JAK-mediated phosphorylation, conformational switch and nuclear translocation $^{21}$ (Figure 2).

Recent work from the O'Shea group further detailed the critical role of IL-15-induced STAT5 target gene expression in NK cells by deleting three of the four Stat5 (Stat5a and Stat5b) alleles 22. This report confirmed the dominant role of STAT5B in maintaining peripheral NK cells as Stat $5 a^{-/}$Stat $5 b^{-/+}$mice possessed significantly more NK cells than $S t a t 5 a^{-/+}$Stat $5 b^{-/}$mice, and revealed that $S t a t 5 a^{-/+}$Stat $5 b^{-/-}$NK cells had a clear reduction in BCL2, IFN- $\gamma$ and T-Bet encoded 
by STAT5 target genes Bcl2, Ifng and $T b x 21^{22}$ (Figure 2). Although some NK cells were able to develop in mice possessing only one Stat5 allele, the population displayed an immature (CD11b KLRG1') phenotype suggesting that their reduced sensitivity to IL-15 couldn't permit appropriate maturation. Although the study did not look at whether a stronger IL-15 signal (e.g. injecting IL-15 into these mutant mice) could partly compensate for Stat5a haploinsufficiency in Stat $5 b^{-/}$mice (by activating a greater proportion of the Stat5a pool), it is clear that NK cell homeostasis is absolutely dependent upon sufficient Stat5 gene expression.

STAT5 has a dynamic array of binding partners that is tuned by the extent of the IL-15 signalling The expression of STAT5 target genes varies greatly depending on the strength of the IL-15 signal (Figure 1). Using CHIP-seq, Villarino et al. found that only about a quarter of the STAT5 binding peaks observed in in vitro IL-15 stimulated NK cells were also present in NK cells under homeostatic conditions (fresh ex vivo NK cells). The authors also demonstrated that this homeostatic STAT5 CHIP-seq 'fingerprint' can be rapidly altered after IL-15 stimulation - after only 2 hours of IL-15 exposure, around 50\% more STAT5-bound genes could be detected in freshly-isolated NK cells (Figure 1). This clearly indicates that the level of IL-15 in vivo is far below that which is able to cause a maximal transcriptional response. Though the authors did not perform similar analysis on NK cells that had been purified and deprived of IL-15 for 2 hours, it would be interesting to see whether withdrawal from homeostatic levels of IL-15 resulted in such dynamic changes in STAT5-bound genes.

One reason that the kinetics of STAT5-mediated gene expression could differ between homeostatic and experimental IL-15 concentrations is the induction of negative regulators of the pathway. Indeed, the negative regulator of IL-15 signalling and emblematic STAT5 target gene Cish (cytokine-induced SH2-containing protein) ${ }^{23}$ was not bound to STAT5 under homeostatic conditions, but presented with numerous, intense STAT5 ChIP-seq peaks after acute IL-15 exposure NK cells (Figure 1) ${ }^{22}$. This is consistent with our earlier work where we failed to detect any alterations in gene expression in ex vivo Cish $^{-1-} \mathrm{NK}$ cells compared to $\mathrm{Cish}^{+/+} \mathrm{NK}$ cells, however we detected over 1000 differentially expressed genes following acute IL-15 stimulation in vitro ${ }^{24}$.

Under homeostatic conditions STAT5 clearly binds its targets to varying degrees, with Cish being among the lesser preferred. From a biological standpoint, it is tempting to speculate that 
this mechanism has evolved to 'smooth-out' the fluctuations of the IL-15 signal in vivo; as Cish is not induced in vivo, it may allow this homeostatic IL-15 signal to persist within the cell as it traverses the relatively IL-15-poor regions of the body.

$\underline{\text { STAT5 proteins form dimers and tetramers that have distinct affinities for the STAT5 binding }}$ $\underline{\text { motif }}$

The molecular mechanism by which STAT5 shows a range of affinities for its target genes has been an area of great interest. Central to this understanding has been the discovery that STAT5 can bind its target gene in dimeric or tetrameric form. To address the contribution of STAT5 dimers versus tetramers in NK cell biology, Warren Leonard's group generated point mutations in the Stat5 genes preventing STAT5 tetramer formation.

These STAT5 point mutant double knock-in (DKI) mice appeared healthy and STAT5 protein expression was normal ${ }^{25}$. However, DKI mice presented with a $\sim 2$ fold reduction in peripheral NK cells due to a specific reduction in mature $\left(\mathrm{CD} 11 \mathrm{~b}^{+}\right) \mathrm{NK}$ cells ${ }^{26}$. Reduced expression of the STAT5 target Bcl2 in DKI NK cells was proposed to account for the systemic reduction in mature NK cells, as this subset is highly dependent on $B c l 2$ and also less responsive to IL-15 given lower surface expression of IL-2R ${ }^{2,23,27}$. Similarly, mature KLRG1 ${ }^{+}$NK cells express lower levels of the STAT5 targets Il2ra, Bcl2 and Socs 2 and cycle slower compared to immature NK cells ${ }^{2}$. The absence of any defect in IL-15 dependent NK cell progenitors (NKP) and immature NK cells in the bone marrow of DKI mice ${ }^{26}$ suggests that the higher expression of the IL-15 receptor complex and increased intrinsic sensitivity to IL-15 by these populations induces adequate STAT5 dimer target gene expression for development. Therefore, the preferential loss of mature NK cells in the DKI mice is consistent with the known variation in IL-15 sensitivity as NK cells develop and mature.

Unsurprisingly, DKI NK cells underwent extensive apoptosis when cultured in sub-optimal IL15 concentrations. However, when cultured in high levels of IL-15 the survival and proliferation characteristics of DKI NK cells were similar to WT NK cells, despite the fact that BCL2 protein levels could never be fully restored. In combination with STAT5 ChIP-seq data that revealed that $B c l 2$ was preferentially bound by STAT5 tetramers ${ }^{26}$, these data clearly demonstrate that STAT5 dimers cannot alone induce the maximal expression of certain target genes. Although the lack of STAT5 tetramer formation can be overridden in vitro with high concentrations of IL-15, the in 
vivo homeostasis of NK cells depends upon STAT5 tetramer formation to drive sufficient Bcl2 expression.

In contrast to this defect in $B c l 2$ expression, it was found that the expression of $M c l 1$, which is absolutely and continuously required for NK cell survival, was normal in DKI NK cells (Figure 2). Thus, $M c l 1$, in contrast to $B c l 2$, is likely to be predominantly bound by STAT5 dimers. Conversely, Cish, which has very little bound STAT5 and mRNA expression under homeostatic conditions ${ }^{22}$, was strongly induced following IL-15 stimulation only in NK cells capable of forming STAT5 tetramers ${ }^{26}$. The importance of a particular IL-15 induced gene to overall NK cell homeostasis therefore appears to directly correlate with its responsiveness to IL-15 through its preference for STAT5 dimers or tetramers. The most crucial genes such as Mcll are sufficiently induced by the minimal STAT5 dimers present under homeostatic conditions. Genes such as Bcl2 that are necessary for the maintenance of a mature NK cell pool require STAT5 tetramers for optimal expression, but can be partly compensated for by STAT5 dimers induced by the low levels of IL-15 in vivo (as evidenced by the fact that mice specifically lacking Bcl2 in NK cells have a greater reduction in NK cell numbers than DKI mice). And finally, genes such as Cish that are only required to dampen the over-stimulation of NK cells are only induced by STAT5 tetramers as a result of high concentrations of IL-15.

\section{Perturbing NK cell homeostasis for cancer treatment - targeting negative regulators of NK cell function}

Given the absolute dependency of NK cells on the IL-15 for their survival and function, this pathway represents one of the prime candidates for the therapeutic manipulation of NK cells for cancer treatment ${ }^{10,11,28-34}$. The simplest way in which this has been achieved has been the administration of recombinant human IL-15 to cancer patients. These trials have observed some promising anti-tumour effects but also resulted in considerable toxicity due to the concomitant expansion of $\mathrm{T}$ cells and cytokine release syndromes. However, the potent ability of IL-15 to expand cytotoxic lymphocytes has meant that there is still considerable industry investment in improving IL-15 therapies to overcome systemic toxicities associated with high-dose IL-15 administration ${ }^{35}$. 
One way that the systemic toxicity of IL-15 administration has been avoided is thorough the development of cellular therapies which use IL-15 a key component of the expansion and activation culture medium. Trials with ex vivo expanded allo- and autologous NK cells have proven to be well tolerated and result in some improved anti-leukaemia responses ${ }^{36}$. However, efficacy of these adoptive NK cell therapies is yet to be proven in larger clinical trials, and ultimately costs must be reduced before such treatments become part of mainstream cancer treatment.

Rather than attempting to directly activate the IL-15 pathway in vivo, a safer and more tolerable approach may be to target the intrinsic negative regulators of NK cell function. Three of the most potent NK cell suppressive mechanisms are discussed below.

\section{CIS}

Cytokine-inducible SH2-containing protein (CIS) is a member of the suppressor of cytokine signalling (SOCS) family of proteins. Unlike many other SOCS family members, mice lacking CIS do not have any overt phenotypic abnormalities and was therefore considered largely redundant. In NK cells exposed to high concentrations of IL-15, Cish is one of the most rapidly induced and highly expressed genes and functions to rapidly dampen the IL-15 signal. Cish expression was shown to be maximally induced at 6-8 hours post-IL-15 stimulation in vitro and regulates IL-15 signalling by binding to phosphorylated JAK1, and inhibiting JAK1 activity via ubiquitination and proteasomal degradation and through inhibition of JAK1 kinase activity ${ }^{24}$ (Figure 2). As a result, $\mathrm{Cish}^{-1-} \mathrm{NK}$ cells have more intense and prolonged JAK1 and STAT5 activation that results in increased proliferation and effector function. However, despite this important function, there was little impact on the NK cell homeostasis in CIS-deficient mice ${ }^{24}$.

Given $\mathrm{Cish}^{-1-}$ mice demonstrate significant resistance to NK cell-dependent tumour growth and metastasis (B16F10 melanoma, E0771 triple negative breast cancer, LWT1 BRAF-mutant melanoma, RM1 prostate carcinoma), it is likely that IL-15 is increased in these tumour microenvironments to levels that induces Cish and in turn limits NK cell function. Indeed, we have observed that CIS expression is higher in NK cells isolated form primary E0771 tumours than NK cells isolated from the non-tumour-bearing contralateral mammary gland (unpublished data). Similarly, we have detected higher levels of STAT5-target gene products Granzyme-B and IFN- $\gamma$ in NK cells from tumour bearing $\mathrm{Cish}^{-/-}$mice compared to controls. 
The role for CIS in NK cells therefore appears to be mainly that of a 'safety net' to prevent the excessive NK cell activation. However, because systemic IL-15 levels are invariably low and only increased in localised sites of inflammation, CIS acts to limit the anti-tumour function of NK cells where it is needed most. The striking increase in effector function seen in CIS-deficient NK cells, together with lack of pathology in CIS-deficient mice, highlights CIS as a highly attractive target for cancer immunotherapy.

\section{$\underline{\mathrm{IL}-18 \& \mathrm{IL}-1 \mathrm{R} 8}$}

IL-18 is a member of the IL-1 family of cytokines and has been studied in detail in regard to its ability to augment NK cell proliferation and effector function however, its role in NK cell homeostasis is less well described. IL-18 is produced as an inactive precursor that requires cleavage by caspase- 1 into its active form. Furthermore, IL-18 activity is balanced by the presence IL-18 binding protein (IL-18BP) which is constitutively present in excess and sequesters free IL-18 due to its high-affinity for the free cytokine ${ }^{37}$. Although IL-18 receptor complex is highly expressed at all stages of NK cell development, the effect of IL-18 alone on NK cell activation is typically weak. In combination, IL-18 can further enhance IL-15 induced NK cell proliferation ${ }^{38}$, whereas and IL-18 synergizes strongly with IL-12 to drive NK cell effector functions, particularly IFN- $\gamma$ production ${ }^{39,40}$. Recently, $I l 18 b p^{-/-}$mice were reported to have an accumulation of immature $\left(\mathrm{CD}_{11 \mathrm{~b}}{ }^{-}\right)$and reduction in mature $\mathrm{KLRG}^{+} \mathrm{NK}$ cells ${ }^{41}$, whereas $I l 18^{-/-}$mice displayed a normal NK cell maturation, consistent with an earlier report ${ }^{42}$. This data suggests that the steady-state levels of IL-18 are not sufficient impact on NK cell homeostasis due the excess of IL-18BP, however, an increase in IL-18 via loss of IL-18BP appears to impact on NK cell differentiation.

IL-1R8 has a single IgG domain and is thought to act as a negative regulator of interleukin-1 receptor family members (ILRs) and toll-like receptors (TLRs) ${ }^{43}$, and act as a decoy receptor for IL-18 ${ }^{37}$. A recent high-profile report has shed light on the role of IL-1R8 in NK cell homeostasis and anti-tumour responses. Molgora et. al. reported high IL-1R8 expression by NK cells compared to other immune cells with expression increased during differentiation. $I l 1 r 8^{-/}$mice were generated and presented with a clear skewing in NK cell maturation towards a more mature KLRG ${ }^{+} \mathrm{NK}$ cells in peripheral organs ${ }^{44}$. This NK cell phenotype contrasts that of the $I l 18 b p^{-/}$mice which is hard to reconcile since both $I l 18 b p^{-/-}$and $I l 1 r 8^{-/} \mathrm{NK}$ cells would 
presumably be exposed to higher levels of IL-18 in vivo. $I l 1 r 8^{-/}$NK cells displayed hyperresponsiveness to IL-18 in vitro with higher levels of intracellular IFN- $\gamma$ and Granzyme B and more phospho-S6 and phospho-IRAK4 compared to control NK cells. These data suggest that IL-1R8 might contribute to NK cell differentiation and act as a negative regulator of IL-18 mediated NK cell activation in vivo.

Although $111 r^{-1-}$ mice showed an accumulation of mature NK cells that are classically less cytotoxic than immature NK cells, it is difficult to predict whether the increased IL-18 sensitivity could compensate or even enhance their NK cell function. However, consistent with their hyperresponsiveness to IL-18, NK cells from $1 l 1 r 8^{-/-}$mice displayed a superior anti-tumour effector function. This included $111 r 8^{-/-}$mice being better able to resist (in comparison to WT mice) the onset and metastasis of a DEN-induced liver cancer model, the intravenous transfer of an MCA fibroscaroma cells or intrasplenic injection of MC38 colorectal carcinoma cell line.

NK cell frequency was elevated in the liver of $111 r 8^{-/-}$mice with a higher proportion of liver-resident NK cells expressing IFN- $\gamma$ suggesting elevated IL-18 in the DEN-induced tumour microenvironment. In contrast, subcutaneous MCA fibrosarcoma grew at the same rate in $1 l 1 r 8^{-/-}$ as control mice supporting a preferential role for NK cells in controlling metastatsis ${ }^{45}$. Taken together, these data suggest that like IL-15, IL-18 levels produced by tumours or infiltrating immune cells could impact overall survival and recurrence free survival in certain cancers. In line with this, IL-18 is used in multiple in vitro NK cell cultivation protocols for adoptive NK cell therapies and has been found to promote the persistence and activity of donor NK cells ${ }^{46}$.

\section{$\underline{\text { TGF- } \beta \text { and adenosine }}$}

Transforming growth factor beta (TGF- $\beta$ ) is a cytokine that signals by phosphorylation of SMAD2/3 as downstream through a heteromeric complex formed by TGF- $\beta$ RII and TGF- $\beta$ RI. This signalling has been extensively studied in cancer cells for its potential to induce epithelial to mesenchymal transition (EMT), promoting tumour cell differentiation, survival, migration, and metastasis to distant organs ${ }^{47}$. In immune cells, this signalling pathway has been revealed as an epigenetic modifier that directly contributes to the differentiation and regulation of several immune cell subsets, such as macrophages ${ }^{48}$, Treg cells ${ }^{49}$, Th17 cells ${ }^{50}$, dendritic cells ${ }^{51}$. Unlike the cell types just mentioned, the conditional deletion of TGF- $\beta$ RII in NK cells was shown not affect their development, maturation or homeostasis. However, when expression is 
upregulated, TGF- $\beta$ plays a major role in suppressing the mTOR metabolic pathway in NK cells and suppresses their ability to kill tumour cells in vitro and in vivo ${ }^{52}$. In addition, we and others also observed that elevated TGF- $\beta$ signalling acts as to re-program NK cell differentiation by upregulating features of Innate Lymphoid Cell (ILC)1-like subsets (e.g. TRAIL and CD49a) whilst simultaneously downregulating genes that distinguish NK cells (e.g. Eomes). This TGF- $\beta$ mediated conversion of NK cells to ILC-1-like cells significantly abrogated their capacity to respond in cancer and infection ${ }^{53,54}$. Taken together, TGF- $\beta$ plays a minor role in NK cell homeostasis, but accumulation of TGF- $\beta$ at sites of infection/tumour formation is able to suppress NK cell function through diverse mechanisms. Interestingly, Cortez and colleagues also described that this ILC1-like differentiation during MCMV virus is also accompanied by expression of the surface ectoenzymes CD39 and CD73, which subsequently hydrolyzes extracellular ATP to adenosine ${ }^{53}$. Although the role of CD39 and CD73 in these ILC1-like cells during MCMV infection is still to be deciphered, these pathways have been extensively studied and targeted in immunotherapy. The tumour microenvironment takes advantage of the immunosuppressive effects of adenosine and it's receptor ADORA2A (A2AR) in immune cells such as NK cells ${ }^{55}$. A2AR was previously shown to reduce NK cell cytotoxic functions and capacity to control metastases upon adenosine binding ${ }^{56}$. More recently, our group also demonstrated that a lack of A2AR expression skews NK cell maturation toward a more mature phenotype under normal conditions ${ }^{57}$. Therefore, there appears to be a role for tonic adenosine signalling in the homeostasis of the NK cell population, but increased levels of adenosine in the tumour microenvironment potently suppresses their function.

The discovery that the STAT5 target CIS as well as IL-1R8, TGF- $\beta$ and adenosine all function to inhibit NK cell activity in either steady-state or in the tumour microenvironment has added several new members to the NK cell checkpoint family. The clinical observation that solid tumours are often richly infiltrated with T cells but poorly infiltrated with NK cells suggests that tumours have evolved several mechanisms to prevent NK cell infiltration, accumulation and effector function. The quest now is to understand the dominant pathways of NK cell suppression in various tumour types to design a range of novel therapeutic inhibitors to complement current NK cell immunotherapy approaches to cancer ${ }^{45}$. 


\section{ACKNOWLEDGEMENTS}

This work is supported project grants from the National Health and Medical Research Council (NHMRC) of Australia (1049407, 1066770, 1057852, 1027472 to N.D.H), an NHMRC CDF2 fellowship (NDH) as well as an NHMRC Independent Research Institute Infrastructure Support scheme grant and a Victorian State Government Operational Infrastructure Scheme grant. NDH is a recipient of research grants from the following organisations: Ian Potter Foundation (AUS), Tour De Cure (AUS), Harry J Lloyd Charitable Trust (USA), Melanoma Research Alliance (USA) and Cancer Research Institute (USA).

\section{CONFLICT OF INTEREST}

NDH and JR are co-founders of oNKo-innate.

Figure 1. STAT5-dependent control of NK cells. STAT5 ChIPseq data generated in ${ }^{22}$ was analysed for STAT5 binding to the indicated genes in resting (ex vivo) and activated (high IL-15 in vitro) NK cells. The upper panel shows examples of STAT5-target genes essential for NK cell homeostasis (Mcl1, Bcl2, Id2, STAT5b) and lineage defining (Ncr1) and as such are maintained at a near maximal level in healthy mice. The bottom panel shows examples of genes critical for NK cell effector function (Prfl, Gzmb) and genes that limit immune responses (Cish, Socs1, Socs 3 ) and as such are potently induced by a local increase in IL-15 concentrations such as during inflammation/cancer/infections.

Figure 2. IL-15 tunes NK cell function through various STAT5 species. IL-15 transduces its signal through the IL-15R $\beta / \gamma$ complex by activating a classical JAK/STAT signalling cascade. STAT5 is the most crucial STAT family member for NK cell function and homeostasis. The two STAT5 isoforms exist in homo- or hetero-dimeric form, which can in turn coalesce to form tetramers. Both STAT5 isoforms play non-redundant roles in maintaining NK cell fitness and function; the presence of only STAT5a is incongruous with NK cell function as evidenced by a dramatic loss of NK cells in Stat5 $a^{-/+}{\text {Stat } 5 b^{-/}}^{-1}$ mice. STAT5b is the minimum essential isoform required for the development and maintenance of NK cells, though Stat $5 a^{-/}$Stat $5 b^{-/+}$mice still have fewer NK cells than WT mice. Optimal NK cell function also requires STAT5 tetramer formation as mice that cannot form STAT5 tetramers present with a 2-fold reduction of NK cells. 
The degree to which STAT5 dimers and tetramers are utilised in vivo also depends on the strength of the IL-15 signal, with elevated levels of IL-15 resulting in greater tetramer formation and the expression of negative-regulators proteins such as CIS.

\section{REFERENCES}

1. Seillet $\mathrm{C}$, Huntington ND, Gangatirkar $\mathrm{P}$ et al. Differential requirement for $\mathrm{Nfil3}$ during NK cell development. J Immunol 2014; 192: 2667-2676.

2. Delconte RB, Shi W, Sathe P et al. The Helix-Loop-Helix Protein ID2 Governs NK Cell Fate by Tuning Their Sensitivity to Interleukin-15. Immunity 2016; 44: 103-115.

3. Seillet C, Belz GT, Huntington ND. Development, Homeostasis, and Heterogeneity of NK Cells and ILC1. Curr Top Microbiol Immunol 2016; 395: 37-61.

4. Yokota Y, Mansouri A, Mori S et al. Development of peripheral lymphoid organs and natural killer cells depends on the helix-loop-helix inhibitor Id2. Nature 1999; 397: 702706.

5. Gascoyne DM, Long E, Veiga-Fernandes $\mathrm{H}$ et al. The basic leucine zipper transcription factor E4BP4 is essential for natural killer cell development. Nat Immunol 2009; 10: $1118-1124$.

6. Boos MD, Yokota Y, Eberl G et al. Mature natural killer cell and lymphoid tissueinducing cell development requires Id2-mediated suppression of E protein activity. $J$ Exp Med 2007; 204: 1119-1130.

7. Carotta S, Pang SH, Nutt SL et al. Identification of the earliest NK-cell precursor in the mouse BM. Blood 2011; 117: 5449-5452. 
8. Lodolce JP, Boone DL, Chai S et al. IL-15 receptor maintains lymphoid homeostasis by supporting lymphocyte homing and proliferation. Immunity 1998; 9: 669-676.

9. Ma A, Koka R, Burkett P. Diverse functions of IL-2, IL-15, and IL-7 in lymphoid homeostasis. Апnи Rev Immunol 2006; 24: 657-679.

10. Burkett PR, Koka R, Chien M et al. Coordinate expression and trans presentation of interleukin (IL)-15Ralpha and IL-15 supports natural killer cell and memory CD8+ T cell homeostasis. J Exp Med 2004; 200: 825-834.

11. Schluns KS, Nowak EC, Cabrera-Hernandez A et al. Distinct cell types control lymphoid subset development by means of IL-15 and IL-15 receptor alpha expression. Proc Natl Acad Sci U S A 2004; 101: 5616-5621.

12. Lodolce JP, Burkett PR, Boone DL et al. T cell-independent interleukin 15Ralpha signals are required for bystander proliferation. J Exp Med 2001; 194: 1187-1194.

13. Fehniger TA, Suzuki K, Ponnappan A et al. Fatal leukemia in interleukin 15 transgenic mice follows early expansions in natural killer and memory phenotype CD8+ T cells. $J$ Exp Med 2001; 193: 219-231.

14. Mlecnik B, Bindea G, Angell HK et al. Functional network pipeline reveals genetic determinants associated with in situ lymphocyte proliferation and survival of cancer patients. Sci Transl Med 2014; 6: 228ra237.

15. Waldmann TA, Tagaya Y. The multifaceted regulation of interleukin-15 expression and the role of this cytokine in NK cell differentiation and host response to intracellular pathogens. Аппи Rev Immunol 1999; 17: 19-49. 
16. Giri JG, Kumaki S, Ahdieh M et al. Identification and cloning of a novel IL-15 binding protein that is structurally related to the alpha chain of the IL-2 receptor. EMBO $J 1995$; 14: 3654-3663.

17. Giri JG, Ahdieh M, Eisenman J et al. Utilization of the beta and gamma chains of the IL2 receptor by the novel cytokine IL-15. EMBO J 1994; 13: 2822-2830.

18. Colpitts SL, Stoklasek TA, Plumlee CR et al. Cutting edge: the role of IFN-alpha receptor and MyD88 signaling in induction of IL-15 expression in vivo. J Immunol 2012; 188: $2483-2487$.

19. Colpitts SL, Stonier SW, Stoklasek TA et al. Transcriptional regulation of IL-15 expression during hematopoiesis. J Immunol 2013; 191: 3017-3024.

20. Wang X, Lupardus P, Laporte SL et al. Structural biology of shared cytokine receptors. Annu Rev Immunol 2009; 27: 29-60.

21. Imada K, Leonard WJ. The Jak-STAT pathway. Mol Immunol 2000; 37: 1-11.

22. Villarino AV, Sciume G, Davis FP et al. Subset- and tissue-defined STAT5 thresholds control homeostasis and function of innate lymphoid cells. J Exp Med 2017; 214: 29993014.

23. Sathe P, Delconte RB, Souza-Fonseca-Guimaraes F et al. Innate immunodeficiency following genetic ablation of Mcl1 in natural killer cells. Nat Commun 2014; 5: 4539.

24. Delconte RB, Kolesnik TB, Dagley LF et al. CIS is a potent checkpoint in NK cellmediated tumor immunity. Nat Immunol 2016; 17: 816-824.

25. Lin JX, Li P, Liu D et al. Critical Role of STAT5 transcription factor tetramerization for cytokine responses and normal immune function. Immunity 2012; 36: 586-599. 
26. Lin JX, Du N, Li P et al. Critical functions for STAT5 tetramers in the maturation and survival of natural killer cells. Nat Commun 2017; 8: 1320.

27. Viant C, Guia S, Hennessy RJ et al. Cell cycle progression dictates the requirement for BCL2 in natural killer cell survival. J Exp Med 2017; 214: 491-510.

28. Huntington ND. The unconventional expression of IL-15 and its role in NK cell homeostasis. Immunol Cell Biol 2014; 92: 210-213.

29. Dubois S, Mariner J, Waldmann TA et al. IL-15Ralpha recycles and presents IL-15 In trans to neighboring cells. Immunity 2002; 17: 537-547.

30. Cooper MA, Bush JE, Fehniger TA et al. In vivo evidence for a dependence on interleukin 15 for survival of natural killer cells. Blood 2002; 100: 3633-3638.

31. Koka R, Burkett PR, Chien M et al. Interleukin (IL)-15R[alpha]-deficient natural killer cells survive in normal but not IL-15R[alpha]-deficient mice. J Exp Med 2003; 197: 977984.

32. Sandau MM, Schluns KS, Lefrancois L et al. Cutting edge: transpresentation of IL-15 by bone marrow-derived cells necessitates expression of IL-15 and IL-15R alpha by the same cells. J Immunol 2004; 173: 6537-6541.

33. Kennedy MK, Glaccum M, Brown SN et al. Reversible defects in natural killer and memory CD8 T cell lineages in interleukin 15-deficient mice. J Exp Med 2000; 191: 771780.

34. Castillo EF, Acero LF, Stonier SW et al. Thymic and peripheral microenvironments differentially mediate development and maturation of iNKT cells by IL-15 transpresentation. Blood 2010; 116: 2494-2503.

This article is protected by copyright. All rights reserved 
35. Waldmann TA. Interleukin-15 in the treatment of cancer. Expert Rev Clin Immunol 2014; 10: $1689-1701$.

36. Mehta RS, Randolph B, Daher M et al. NK cell therapy for hematologic malignancies. Int J Hematol 2018.

37. Dinarello CA, Novick D, Kim S et al. Interleukin-18 and IL-18 binding protein. Front Immunol 2013; 4: 289.

38. French AR, Holroyd EB, Yang L et al. IL-18 acts synergistically with IL-15 in stimulating natural killer cell proliferation. Cytokine 2006; 35: 229-234.

39. Chaix J, Tessmer MS, Hoebe K et al. Cutting edge: Priming of NK cells by IL-18. $J$ Immunol 2008; 181: 1627-1631.

40. Lauwerys BR, Renauld JC, Houssiau FA. Synergistic proliferation and activation of natural killer cells by interleukin 12 and interleukin 18. Cytokine 1999; 11: 822-830.

41. Harms RZ, Creer AJ, Lorenzo-Arteaga KM et al. Interleukin (IL)-18 Binding Protein Deficiency Disrupts Natural Killer Cell Maturation and Diminishes Circulating IL-18. Front Immunol 2017; 8: 1020.

42. Huntington ND, Tabarias H, Fairfax K et al. NK cell maturation and peripheral homeostasis is associated with KLRG1 up-regulation. J Immunol 2007; 178: 4764-4770.

43. Molgora M, Barajon I, Mantovani A et al. Regulatory Role of IL-1R8 in Immunity and Disease. Front Immunol 2016; 7: 149.

44. Molgora M, Bonavita E, Ponzetta A et al. IL-1R8 is a checkpoint in NK cells regulating anti-tumour and anti-viral activity. Nature 2017; 551: 110-114. 
45. Guillerey C, Huntington ND, Smyth MJ. Targeting natural killer cells in cancer immunotherapy. Nat Immunol 2016; 17: 1025-1036.

46. Ni J, Miller M, Stojanovic A et al. Sustained effector function of IL-12/15/18preactivated NK cells against established tumors. J Exp Med 2012; 209: 2351-2365.

47. $\mathrm{XuJ}$, Lamouille S, Derynck R. TGF-beta-induced epithelial to mesenchymal transition. Cell Res 2009; 19: 156-172.

48. Sugiyama Y, Kakoi K, Kimura A et al. Smad2 and Smad3 are redundantly essential for the suppression of iNOS synthesis in macrophages by regulating IRF3 and STAT1 pathways. Int Immunol 2012; 24: 253-265.

49. Takimoto T, Wakabayashi Y, Sekiya T et al. Smad2 and Smad3 are redundantly essential for the TGF-beta-mediated regulation of regulatory T plasticity and Th1 development. $J$ Immunol 2010; 185: 842-855.

50. Ichiyama K, Sekiya T, Inoue N et al. Transcription factor Smad-independent T helper 17 cell induction by transforming-growth factor-beta is mediated by suppression of eomesodermin. Immunity 2011; 34: 741-754.

51. Kashiwagi I, Morita R, Schichita T et al. Smad2 and Smad3 Inversely Regulate TGF-beta Autoinduction in Clostridium butyricum-Activated Dendritic Cells. Immunity 2015; 43: $65-79$.

52. Viel S, Marcais A, Guimaraes FS et al. TGF-beta inhibits the activation and functions of NK cells by repressing the mTOR pathway. Sci Signal 2016; 9: ra19. 
53. Cortez VS, Cervantes-Barragan L, Robinette ML et al. Transforming Growth Factor-beta Signaling Guides the Differentiation of Innate Lymphoid Cells in Salivary Glands. Immunity 2016; 44: 1127-1139.

54. Gao Y, Souza-Fonseca-Guimaraes F, Bald $\mathrm{T}$ et al. Tumor immunoevasion by the conversion of effector NK cells into type 1 innate lymphoid cells. Nat Immunol 2017; 18: 1004-1015.

55. Beavis PA, Milenkovski N, Stagg J et al. A2A blockade enhances anti-metastatic immune responses. Oncoimmunology 2013; 2: e26705.

56. Beavis PA, Divisekera U, Paget C et al. Blockade of A2A receptors potently suppresses the metastasis of CD73+ tumors. Proc Natl Acad Sci U S A 2013; 110: 14711-14716.

57. Young A, Ngiow SF, Gao Y et al. A2AR adenosine signaling suppresses natural killer cell maturation in the tumor microenvironment. Cancer Res 2017.
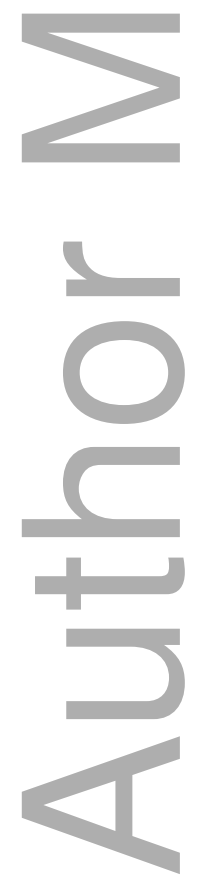


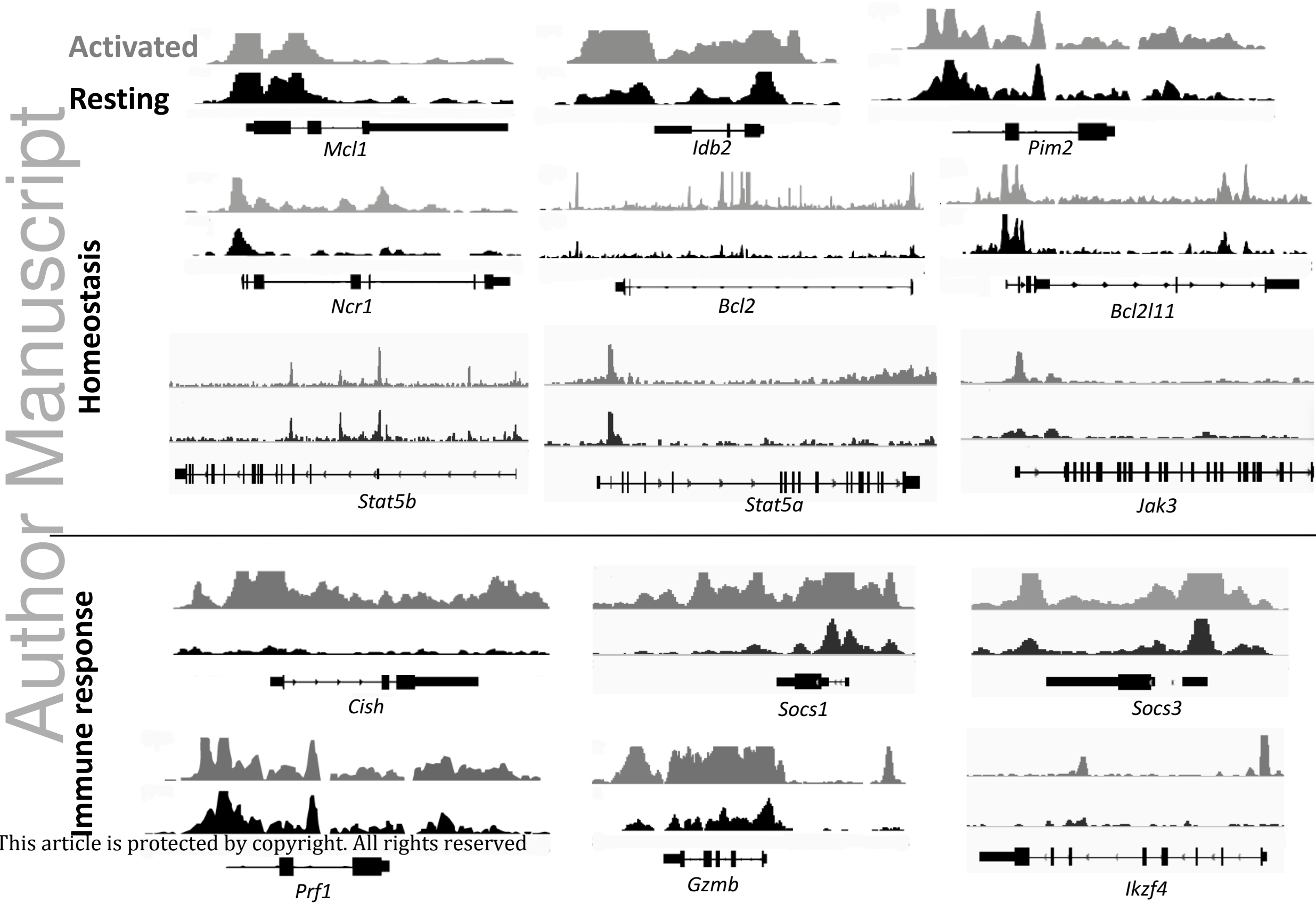




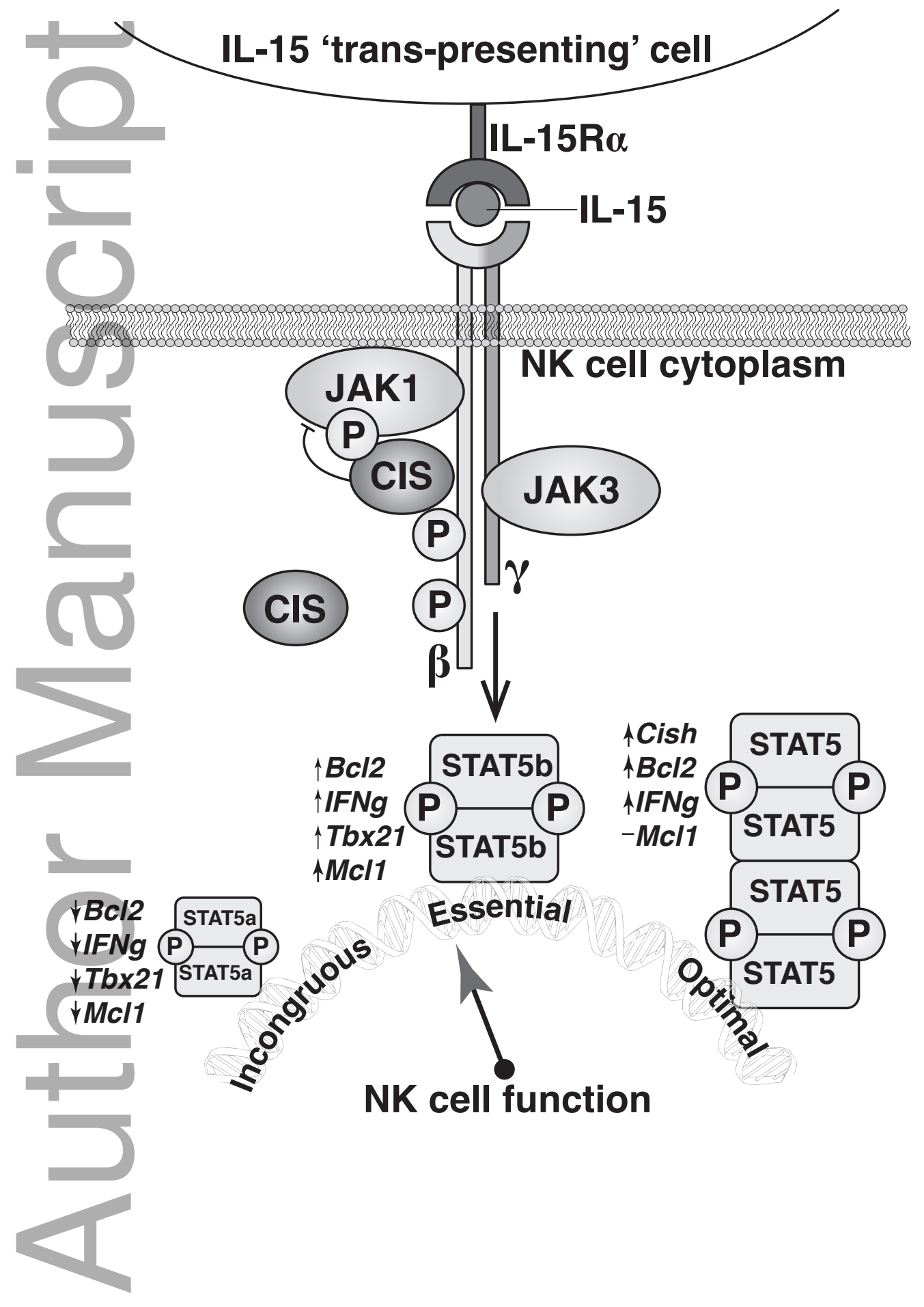




\section{University Library}

\section{- M M N E R VA A gateway to Melbourne's research publications}

Minerva Access is the Institutional Repository of The University of Melbourne

\section{Author/s:}

Rautela, J;Souza-Fonseca-Guimaraes, F;Hediyeh-Zadeh, S;Delconte, RB;Davis, MJ;Huntington, ND

Title:

Molecular insight into targeting the NK cell immune response to cancer

\section{Date:}

2018-05-01

\section{Citation:}

Rautela, J., Souza-Fonseca-Guimaraes, F., Hediyeh-Zadeh, S., Delconte, R. B., Davis, M. J. \& Huntington, N. D. (2018). Molecular insight into targeting the NK cell immune response to cancer. IMMUNOLOGY AND CELL BIOLOGY, 96 (5), pp.477-484. https://doi.org/10.1111/ imcb. 12045 .

Persistent Link:

http://hdl.handle.net/11343/283784 